\title{
Experimental set-up for exciting and detecting Magneto-optical effects and Surface Plasmon Resonance simultaneously
}

\author{
F. Galvez ${ }^{1}$, D. Pérez de Lara ${ }^{2}$, M. A. García ${ }^{3,4}$ and J. L. Vicent ${ }^{1,2}$ \\ ${ }^{1}$ Dpto. de Física de Materiales, Universidad Complutense de Madrid, 28040 Madrid, Spain \\ ${ }^{2}$ IMDEA-Nanociencia, c/ Faraday 9, Cantoblanco, 28049 Madrid, Spain \\ ${ }^{3}$ Instituto de Cerámica y Vidrio, Consejo Superior de Investigaciones Científicas, 28049 Madrid, Spain \\ 4 Instituto de Magnetismo Aplicado 'Salvador Velayos', Universidad Complutense de Madrid, \\ 28230 Madrid, Spain
}

\begin{abstract}
We present here an experimental set-up system to excite and measure simultaneously surface plasmon resonance (SPR) and magneto-optic signal in hybrid magneto-plasmonic systems using two independent light sources. The system can be used to excite and measure both types of SPR, localized surface plasmons in nanostructures and surface plasmon polaritons in thin films. It also allows measuring SPR in presence of magnetic fields and recording magnetooptical hysteresis loops while exciting SPR.
\end{abstract}


Magnetic nanomaterials are key elements of nowadays technological devices including several types of sensors and actuators $[1,2,3,4]$. Similarly, the interest for plasmonic materials, that allows the manipulation of light at sub-wavelength scale, has grown drastically in the last years, and applications have been developed in the fields of optical communications, environmental sensors and biomedicine $[5,6]$.

The combination of ferromagnetic and plasmonic elements in a single device results appealing. Besides the interesting properties of both elements, proximity and coupling effects lead to additional effects, as the modulation of light signals via magnetic fields or the modulation of the magnetic properties via plasmons excitation, that is the basis of magneto-plasmonic sensors $[7,8]$. Thus, in the last years the field of magneto-plasmonics has received increasing attention becoming a very active field in condensed matter physics.

One of the most interesting effects in those systems is the coupling between magneto-optical properties and surface plasmon excitation $[9,10]$. The dielectric tensor of a ferromagnetic element depends on its magnetization state so the optical and plasmonic properties can be modified by applying magnetic fields $[11,12]$. This type of effects is commonly studied by measuring the magnetooptical properties of the system using a single light beam that excites SPR and measures the magneto-optical response. These experiments provide information mainly about the modification of the SRP excitation via magnetic fields.

A different kind of experiments that can provide complementary information should be the use of two independent light sources: one for the excitation of SPR and another one for the measurement of magneto-optical properties.

While this approach results experimentally more complicated, it may have some advantages. Using two independent light sources for the surface plasmon excitation and magneto-optical measurement, it is possible to vary parameters of one of the processes while the second remains unaltered, providing key information to understand the coupling phenomena.

Nevertheless, this type of experiments is technologically challenging. Focusing two laser beams at the same point and measuring reflected beams separately 
and with accuracy is not straightforward, especially the magneto-optical signal for which variations are really small.

In addition, we must consider the geometrical difficulties to implement both systems together. Localized surface plasmons (LSP) in nanostructures exhibit a resonance frequency and can be excited and measured by direct illumination of the sample surface and recording the optical absorption spectrum [13]. On the contrary, the excitation of propagating surface plasmon polaritons (SPP) in thin films requires from evanescent fields and more complicated geometries are needed. The most common configuration for the measurement of propagating SPP are those working in the attenuated total reflection conditions (ATR), using a Raether-Kretschmann configuration [14]. This set-up requires coupling the sample to a prism that must be illuminated on the back side and scanning the incidence angle to record SPP spectra. This complicated geometry, in addition to the problems related to the use of two laser beams co-aligned on the sample above described, makes experimentally challenging this type of combined magneto-optical and surface plasmons measurements.

We present here a set-up for the combined measurement of SPR and magnetooptical Kerr effect. The system allows registering magnetization curves and SPR simultaneously as well as combined measurements, for instance, recording magnetization curves while exciting SPR or register SPR spectra applying a variable magnetic field. With the proper geometry and optical system developed here, it is possible to obtain those data with accurate signal to noise ratio in order to study coupling phenomena in magneto-plasmonic systems.

Figure 1 presents a scheme of the system with a configuration arranged for longitudinal Kerr measurements and both types of surface plasmons, LSP and SPP.

For the excitation and measurement of LSP (figure 1a) the sample surface can be illuminated directly. In order to measure the optical absorption spectrum, the sample can be illuminated with white light and use a waveguide and dispersive spectrophotometer to record the transmitted light and obtain the optical absorption spectrum. In our device, we used a CCS200 dispersive spectrometer (Thorlabs) that allows to record spectra in the range 200-1000 nm with $2 \mathrm{~nm}$ 
resolution in few seconds. In the case of opaque samples, the LSP spectrum can be obtained in reflectance mode, placing the waveguide in the front side of the sample at the space between the incident and reflected beams of the Kerr set-up. In both cases, we found unavoidable that the laser used to measure the magneto-optical properties is also detected in the optical spectrum. However, this light is a highly monochromatic laser so it just appears as a single point in the spectrum that can be eliminated manually. Note that, for instance the FWHM of a HeNe laser is of the order of $0.002 \mathrm{~nm}$ while the absorption curves corresponding to LSP have a FWHM of tens of nanometers. For experiments where only excitation of LSP is required and no measurement needed, the light source can be replaced by a laser and the waveguide removed.

The set-up for excitation of SPP, that results more complicated, is presented in figure 1b. The sample, grown onto a transparent substrate is fixed to a silica $(\mathrm{n}=1.52)$ triangular prism (1 cm side) using index matching gel and mounted on a motorized rotation stage. This rotation stage has a three-axis position system with micrometers for a perfect alignment of the sample. Basically, the SPR excitation and measurement are performed on the back side of the samples while the magneto-optical properties are measured in the front one.

The laser for SPR excitation (p-polarization) is mounted onto a support holder with an orientation system to adjust the laser beam direction and, with appropriated optical apertures, to illuminate the sample through the prism side. A photodetector (Si photodiode) records the reflected signal. It is better to use a photodetector separated from the rotation stage since mechanical movement of the wires may induce noise. Then, when the sample rotates an angle $\theta$, the reflected beam changes its direction an angle $2 \cdot \theta$. Therefore, to avoid that the reflected beam moves out of the photodetector while scanning the incidence angle, we must use an elongated photodiode and place it close to the sample. A typical SPR spectrum requires scanning an incidence angle of $\theta \sim 10^{\circ}$, so the reflected beam rotates $2 \theta \sim 20^{\circ}$; if the detector is placed at about $\mathrm{d}=5 \mathrm{~cm}$ of the sample, along the spectrum, the spot will move laterally $2 \cdot \theta \cdot d \sim 1.7 \mathrm{~cm}$, so a detector with a dimension larger than $2 \mathrm{~cm}$ should be appropriated. In our device we use a Silicon photodiode model S2551 from Hamamatsu with $2.9 \mathrm{~cm}$ length. 

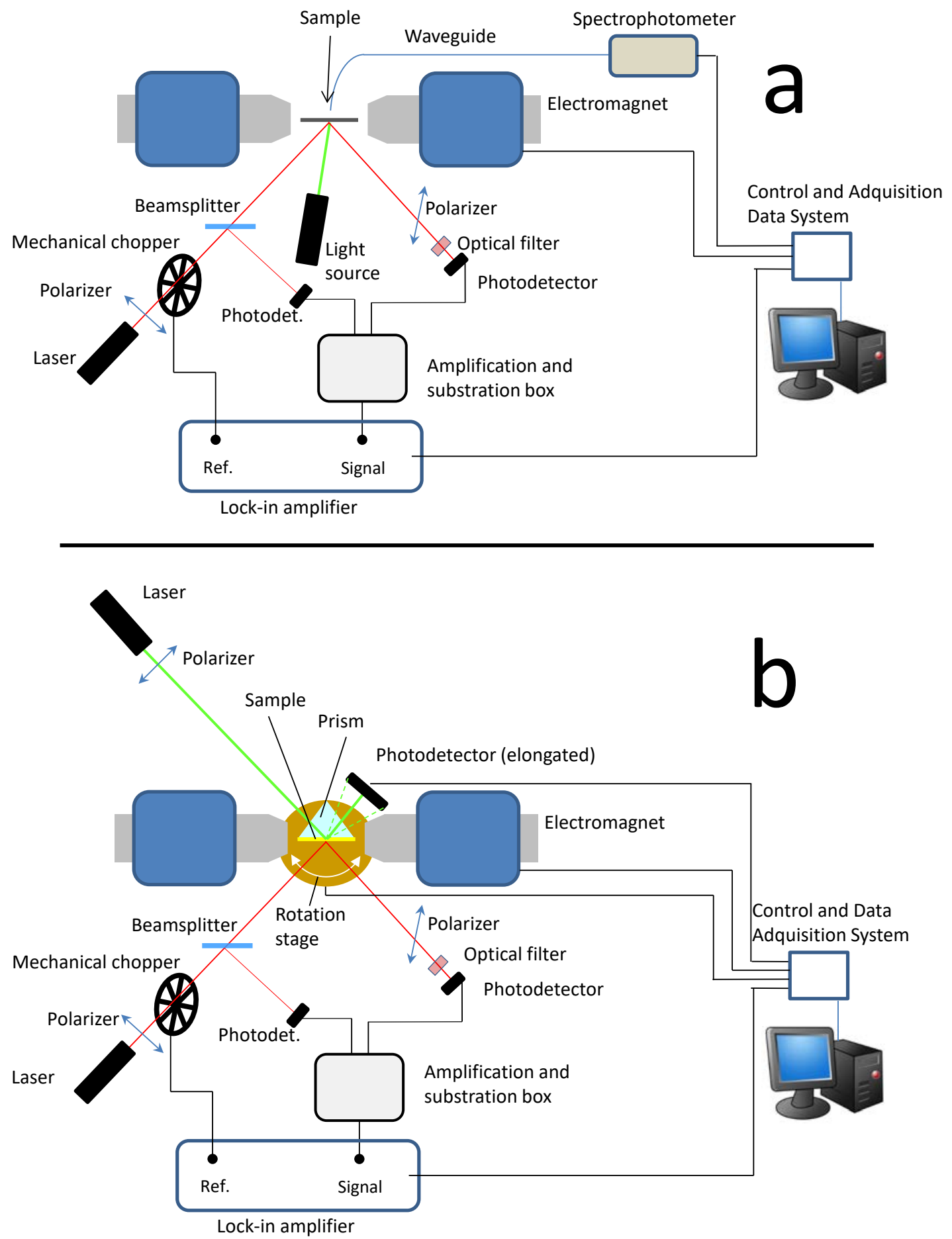

Figure 1. Scheme of the set-up for combined magnetic and SPR measurements. (a) for LSP and (b) for SPP.

As above indicated, we used a triangular prism for the SPR set-up. While triangular prisms ensure a homogenous incidence angle for the whole wavefront, the refraction of the laser beam at the prism surface induces a shift 
of the spot at the sample surface that depends on the incidence angle. In particular, for this type of prism, when scanning the incidence angle $10^{\circ}$ (typical value for a SPR spectrum), the spot will move about $\sim 0.6 \mathrm{~mm}$ [15]. Therefore, when carrying out experiments that involves scanning the incidence angle of the SPR set-up, this issue must be considered to ensure the proper alignment of both beams.

Regarding the magneto-optical properties set-up, we use the same configuration irrespective of the excitation of LSP or SPP. In the longitudinal configuration, we use a solid state laser that can be in $\mathrm{p}$ or $\mathrm{s}$ polarization. Longitudinal Kerr rotation is about equal for both polarizations up to incidence angles of $30^{\circ}$, while for larger angles the rotation is larger for $p$ polarization $[16,17]$, so the latter is preferred when measuring longitudinal Kerr rotation. The reflected beam passes through an analyzer with the axis almost perpendicular to the incidence polarization and reaches a photodetector.

Measuring Kerr signal while exciting SPR with another laser presents several difficulties. Magneto-optical signals of ferromagnetic elements are quite small. For instance, full Kerr rotation for pure bulk Fe is $0.53^{\circ}$, similar values are found for other ferromagnetic metals and alloys [18], and this value is even lower in thin films [16]. Consequently, in order to improve the relative variations, magneto-optical measurements are commonly performed close to extinction situation, i.e., with the analyzer perpendicular to the incident light polarization. Thus, the number of photons reaching the detector of the magneto-optical measurements is small. Additionally, in magneto-plasmonic systems, a part of the reflected light may arise from the plasmonic elements (with very limited magneto-optical activity). Hence, detecting the magneto-optical signal results more difficult. In this situation, the presence of the laser to excite SPR focused on the same point that the laser used to measure magneto-optical signals can induce large noise rending difficult to obtain reliable data with good resolution (note that in order to have significant effects of the SPR excitation on the magnetic properties, the power density of the laser used to excite surface plasmon is usually of $\sim W / \mathrm{cm}^{2}$ ). Since upon excitation of SPR, a significant fraction of the light is scattered $[13,14]$, it results unavoidable that a part of this scattered light exits parallel to the reflected beam of the magneto-optical 
measurements reaching the detector. Nevertheless, we found it is possible to overcome the above indicated problems and obtain good signal to noise ratio combining three procedures in the magneto-optical set-up.

The first one consists in measuring in differential mode to eliminate effects related to laser fluctuations: before the laser of the magneto-optical system beam reaches the sample, a beam splitter is used to deflect a fraction of the light $(\sim 5 \%)$ to a photodiode that is used as reference signal (figure 1 ). The reference and reflected signal are amplified to have similar values and then subtracted electronically with a compensation system.

The second element is to modulate mechanically the laser beam of the magneto-optical system with a mechanical chopper (while excitation of SPR is continuous) and measure the compensated signal with a lock-in amplifier. In this way, even if a part of the laser used to excite SPR (continuous) reaches the detector of the magneto-optical signal, it will be removed from the signal provided by the lock-in amplifier. Modulation frequencies of the order of 70-200 $\mathrm{Hz}$ result appropriated (avoiding multiples of $50 \mathrm{~Hz}$ ).

However, even with this set-up we found some differences in the magnetooptical signal when SPP is excited due to an experimental artifact. The sensitivity of photodetectors is not perfectly linear, so in presence of a continuous light background the response to a modulated signal is different. Therefore, if a fraction of the light for the SPP excitation partially reaches the detector, it may produce a background that makes the photodetector to work in a different part of the response curve, and therefore providing a different response for the same modulated stimulus. This problem can be overcome by placing a narrow band filter in front of the detector of the magneto-optical signal that allows passing the laser beam of the magneto-optical signal blocking that of the SPP excitation. To this purpose, the laser used in the magneto-optical setup and the laser used to excite SPP must have different wavelengths, so the signal from the SSP excitation can be blocked by the filter. In particular, we found that, using a 543 (green) HeNe nm laser for SPR and a $635 \mathrm{~nm}$ (red) laser for magneto-optical measurement and placing a bandpass filter centered at $635 \mathrm{~nm}$ and $10 \mathrm{~nm}$ full-width-at-half-maximum, the problem was fully solved. The whole system is controlled via computer with a home-made software (Visual Basic) that allows to measure magnetization curves, SPR spectra as 
well as the magneto-optic or plasmonic signals when scanning incidence angle or magnetic fields.

In order to illustrate the capabilities of the system we present here measurements of a SPR and magneto-optical Kerr effects on hybrid magnetoplasmonic systems.

Figure 2 shows a SPP spectrum of a $50 \mathrm{~nm} \mathrm{Au}$ film. Details on the sample preparation and geometry have been given elsewhere [19]. The experimental curve matches very well the calculated one confirming that the set-up allows measuring SPP spectra without introducing significant distortions in the curves. Results of SPP measurements with patterned ferromagnetic nanostructures on top of the Au film have been reported elsewhere, exhibiting also good signal to noise-ratio [19].

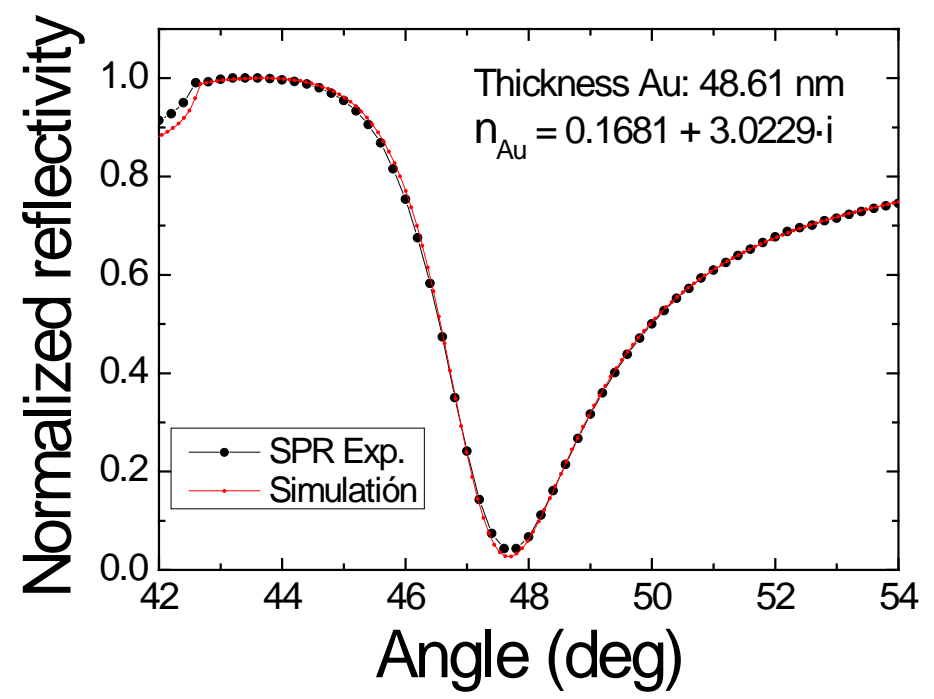

Figure 2. SPP spectra measured with the experimental setup and the fit to a curve calculated according to Fresnel equation using Winspall software [20].

In order to check that there is the system allows to measure MOKE curves with good signal to noise ratio and free of spurious alterations the MOKE signal upon excitation of SPR, we measured MOKE on a sample consisting on an array Permalloy/Au bars $(800 \times 200 \times 50 \mathrm{~nm})$ patterned on a silicon substrate with and without excitation of SPR. This sample exhibit both localized SPR (with resonance at $\lambda \approx 540 \mathrm{~nm}$ ) but no significant magneto-plasmonic coupling effects are expected for this geometry. MOKE was measured in longitudinal 
configuration at the reflected and diffracted beam $(m=1)$ with a $635 \mathrm{~nm}$ diode laser while SPR was excited with a $543 \mathrm{~nm}$ HeNe laser with power density 0.5 $\mathrm{W} / \mathrm{cm}^{2}$. As figure 3 shows, no alteration of the MOKE signal measured in diffracted mode beyond the experimental resolution was observed upon excitation of the SPR. Similar results were found measuring in reflection mode and/or exciting SPP in other sample. These results confirm the capability of the system to measure MOKE with good resolution and free of spurious alteration of MOKE signal due to optical interference with the laser used to excite surface plasmons.

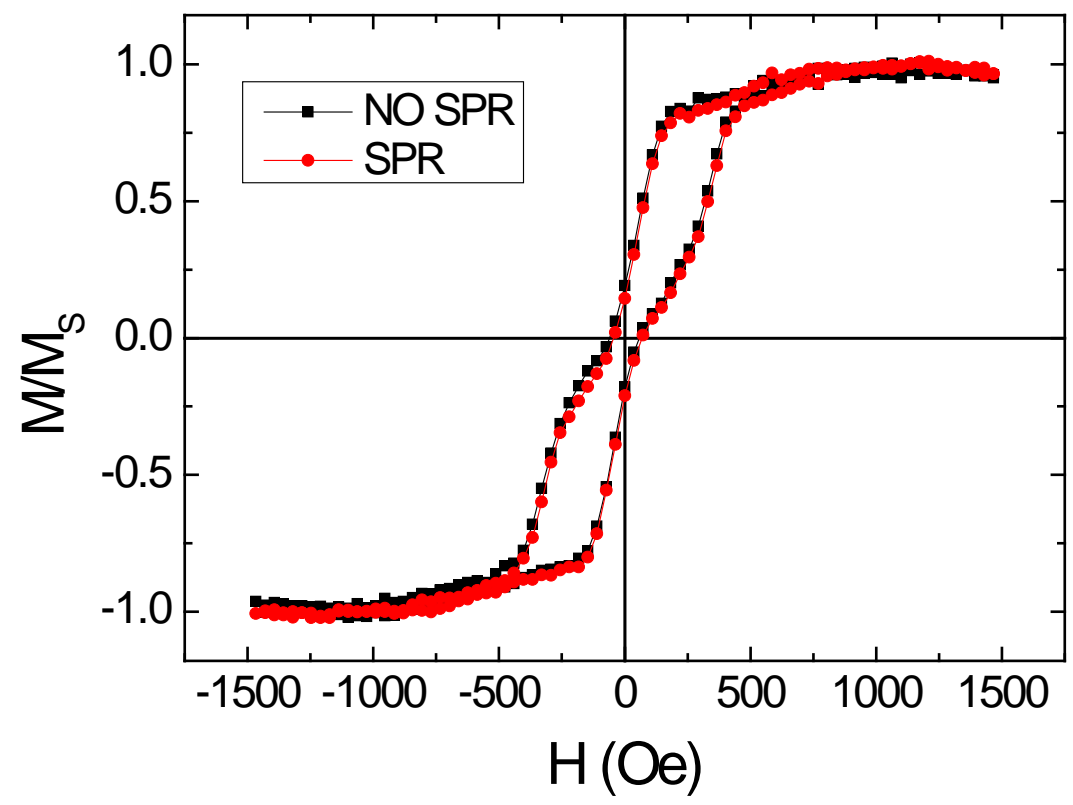

Figure 3. Magnetization curves from the Au+Py bars measured by Kerr technique along the easy axis with and without excitation of SPR.

Depending on the dimensions of the electromagnet, it can be difficult to illuminate the back side of the sample at about $45^{\circ}$ required to excite SPP [14] as shown in figure $4 \mathrm{a}$. In this case, a possible solution is to use a small mirror placed close to the prism in order to reach the proper illumination angle to excite SPP as depicted in figure $4 \mathrm{~b}$. It is essential for the mirror to be mechanically stable and isolated from the electromagnet to avoid vibrations induced by the electromagnet power supply that often propagate through the wires. 


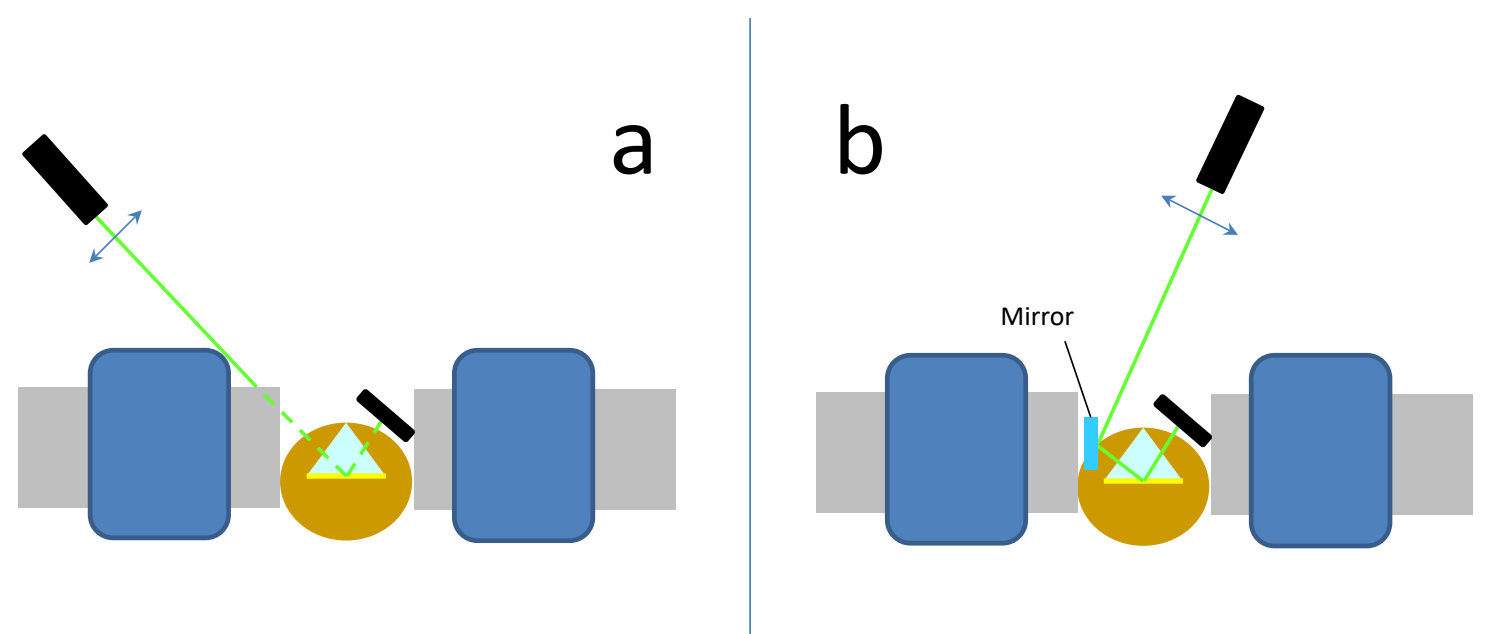

Figure 4. (a) Geometrical problem with the electromagnet that does not avoid to illuminate the sample at the proper angle to excite SPP. (b) Solution placing a mirror close to the sample.

An important issue of the above described system is that, when recording a SPP curve in presence of a magnetic field, the sample rotates, so the relative orientation of the sample and the magnetic field varies, typically $\Delta \theta \approx 10^{\circ}$. This may represent a variation of the component of the magnetic field in the plane of the sample of about $2 \%$. This problem can be solved by using a motorized laser and detector with coupled movement (as in X-Ray diffractometers working $\theta-2 \theta$ configuration). With this arrangement, the sample remains in the same place when scanning the incidence angle and the magnetic field is always parallel to the sample surface.

Placing the electromagnet vertically, the system allows measuring the transversal MOKE signal (figure 5a), that provides information complementary to the longitudinal one [21]. Moreover, with this configuration, it is also possible to measure the longitudinal MOKE signal by illuminating the sample with certain vertical incidence angle and placing the detector above the excitation laser, making the reflection plane to be vertical (see figure 5b). It is also noteworthy that in this configuration of vertical electromagnet the magnetic field is always perpendicular to the wavevector of SPP which may be relevant for the study of magneto-plasmonic interaction effects.

In order to illustrate the resolution of the system with this vertical configuration, figure 6 shows a longitudinal MOKE curve measured with this geometry from $\mathrm{FeCo} / \mathrm{Au} / \mathrm{FeCo}$ trilayer bars patterned on a silicon substrate (samples details 
provided in [22]). The set-up allows recording the magneto-optical signal with resolution good enough to clearly identify the hysteresis loops corresponding to the top and bottom layers.

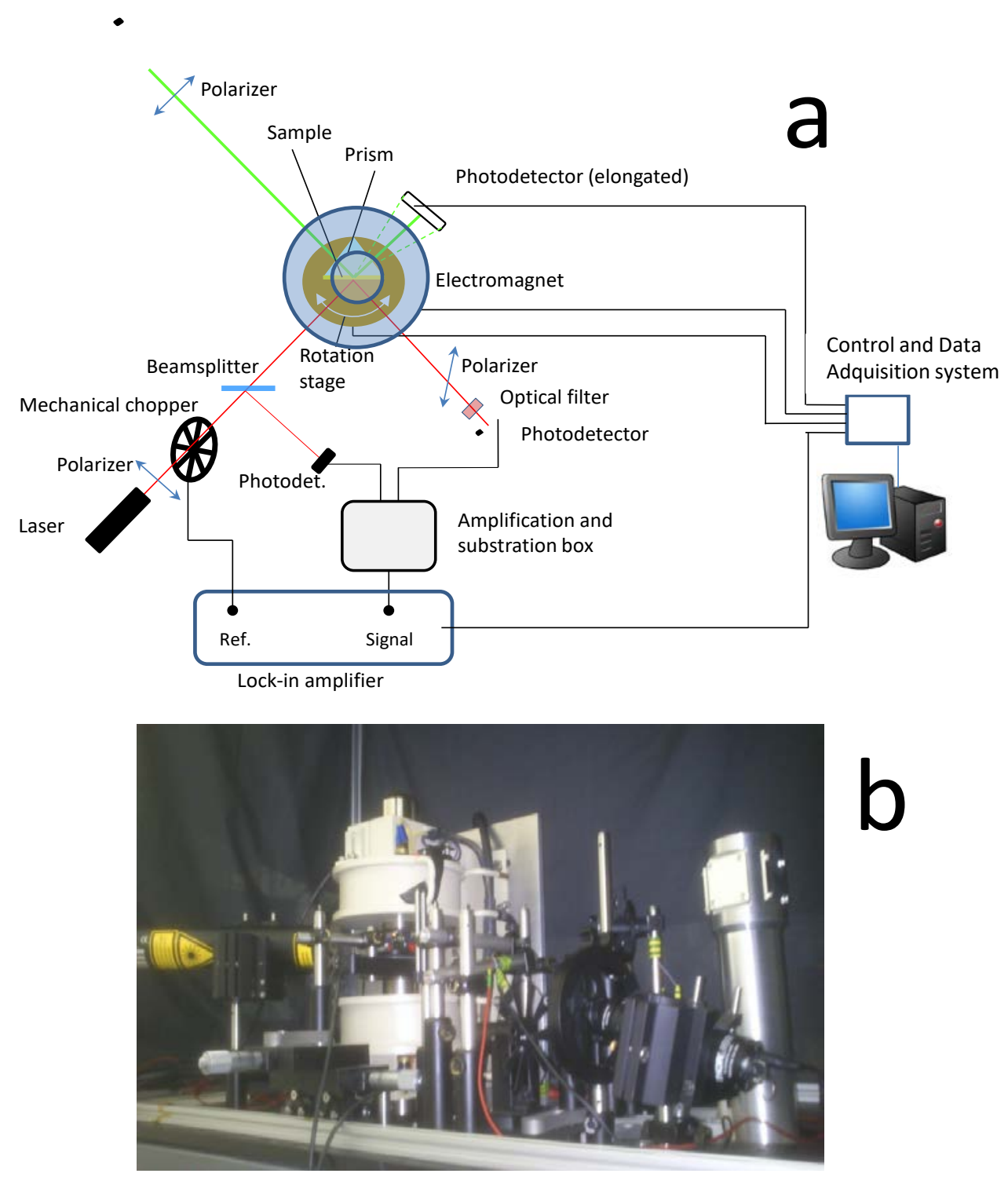

Figure 5. (a) Scheme of the system with the electromagnet in vertical and (b) photo of the system arranged to measure longitudinal Kerr effect in this configuration. 


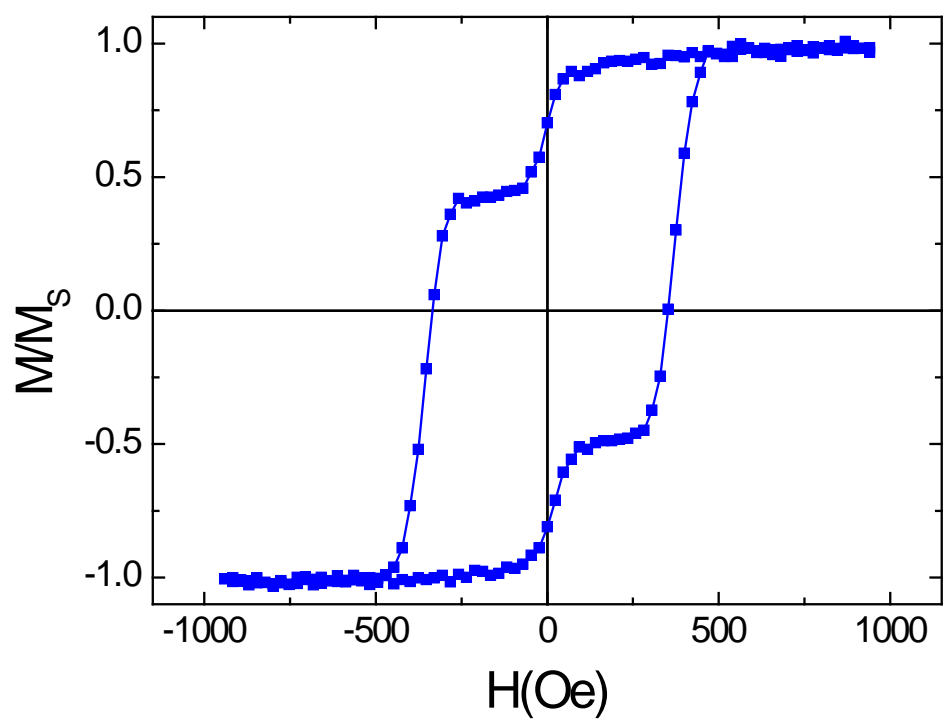

Figure 6. Magnetization curve from patterned $\mathrm{FeCo} / \mathrm{Au} / \mathrm{FeCo}$ trilayers measured in diffraction mode with the described set-up.

In summary we presented here a system that allows the independent excitation and measurement of MOKE signals and SPR (both LSP and SPP) using two light sources. Despite the geometrical complexity to adapt both systems and the difficulties to measure in presence of two optical signals, an appropriate design in combination with the use of modulation, differential mode and wavelength filtering, allows the recording of both SPR and MOKE signal with accuracy. This set-up can provide additional information to study the interaction of SPR and magneto-optical signals for the design of new magneto-optical sensors and actuators.

\section{Acknowledgments}

C. Romero is acknowledged for the design and fabrication of the I-V converter and amplification and compensation system. This work has been supported by the Ministerio Español de Economia y Competitividad (MINECO) MAT2013-48009-C4-1 and FIS2013-45469-C4-1 and Comunidad de Madrid (CM) S2013/MIT-2850. Project supported by a 2016 BBVA Foundation Grant for Researchers and Cultural Creators. M. Abuin is acknowledged for providing the FeCo/Au/FeCo patterned sample. 


\section{References}

[1] A. J. Fleming, A review of nanometer resolution position sensors: Operation and performance Sens. Actuators A-Phys. 190 (2013) 106-126.

[2] S. Parkin, X.Jiang, C.Kaiser, A. Panchula, K.Roche, M. Samant, Magnetically engineered spintronic sensors and memory, Proc. IEEE 91 (5) (2003) 661-680.

[3] A. Persson, R. Bejhed, F. Oesterberg, K. Gunnarsson, H. Nguyen, G. Rizzi, M. F. Hansen, $P$. Svedlindh, Modelling and design of planar Hall effect bridge sensors for low-frequency applications, Sens. Actuators A-Phys. 189 (2013) 459-465.

[4] K. Zhang, L. Zhang, L. Fu, S. Li, H. Chen, Z.-Y. Cheng, Magnetostrictive resonators as sensors and actuators, Sens. Actuators A-Phys. 200 (2013) 2-10.

[5] W. L. Barnes, A. Dereux, T. W. Ebbesen, Surface plasmon subwavelength optics, Nature 424 (2003) 824-830.

[6] J. A. Schuller, E. S. Barnard, W. Cai,Y. Chul Jun, J. S. White, M. L. Brongersma, Plasmonics for extreme light concentration and manipulation, Nature Mat. 9 (2010) 193-204.

[7] G. Armelles, A. Cebollada, A. García-Martín, M. U. González, Magnetoplasmonics: Combining Magnetic and Plasmonic Functionalities, Adv. Opt. Mat. 1 (1) (2013) 10-35.

[8] C. Yan, L. Han, J. Yang, W. Gu, Y. Liao, Magnetic field sensing using evanescent waves in the Kretschmann configuration Sens. Actuators A-Phys. 220 (2014) 85-91.

[9] J. B. González-Díaz, A. García-Martín, J. M. García-Martín, A. Cebollada, G. Armelles, B. Sepúlveda, Y. Alaverdyan and M. Käll, Plasmonic Au/Co/Au Nanosandwiches with Enhanced Magneto-optical Activity, Small 4 (2) (2008) 202-205.

[10] N. Maccaferri, X. Inchausti, A. García-Martín, J. C. Cuevas, D. Tripathy, A. O. Adeyeye, P. Vavassori, Resonant Enhancement of Magneto-Optical Activity Induced by Surface Plasmon Polariton Modes Coupling in 2D Magnetoplasmonic Crystals, ACS Photonics 2 (12) (2015) 1769-1779.

[11] K. Lodewijks, N. Maccaferri, T. Pakizeh, R. K. Dumas, I. Zubritskaya, J. Akerman, P. Vavassori, A. Dmitriev, Magnetoplasmonic Design Rules for Active Magneto-Optics, Nano Lett. 14 (12) (2014) 7207-7214.

[12] G. A. Wurtz, W. Hendren, R. Pollard, R. Atkinson, L. Le Guyader, A. Kirilyuk, T. Rasing, I. I. Smolyaninov and A. V. Zayats, Controlling optical transmission through magneto-plasmonic crystals with an external magnetic field, New J. Phys. 10 (2008) 105012.

[13] U. Kreibig, M. Vollmer, Optical Properties of metal clusters, Springer Series in Materials Science Volume 25 (Springer 1995).

[14] H. Raether, Surface Plasmons on Smooth and Rough Surfaces (Springer 1986).

[15] A. Serrano O. Rodríguez de la Fuente, V. Collado, J. Rubio-Zuazo, C. Monton, G. R. Castro, and M. A. García, Rev. Sci. Instrum 83(2012) 083101.

[16] C-Y. You and S-C. Shin, Derivation of simplified analytic formulae for magneto-optical Kerr effects Appl. Phys. Lett. 69 (9) (1996) 1315.

[17] J. Zak, E. R. Moog, C. Liu, S. Bader, Universal Approach to magneto-optics J. Magn. Magn. Mat. 89 (1990) 107-123.

[18] J. M. D. Coey, Magnetism and Magnetic Materials (Cambridge Univ. Press 2010).

[19] F. Galvez, J. Del Valle A. Gomez, M. R. Osorio D. Granados, D. Perez de Lara, M. A. Garcia and J. L. Vicent, Plasmonic nanodevice with magnetic funcionalities: fabrication and characterization, Opt. Mat. Exp. 6 (10) (2016) 3086-3096.

[20] We simulated the spectra with the WINSPALL freeware code, see http://www.mpipmainz.mpg.de/knoll/soft/ for WINSPALL freeware code. 
[21] C. Penfold, R T Collins, A P B Tufaile, Y. Souche, Transverse magneto-optical Kerr effect:the phase change of reflected light J. Magn. Magn. Mat. 242-245 (2002) 964.

[22] M. Abuin, M. Maicas, M. A. García, L. Pérez, A. Mascaraque, Tailoring the magnetization states in 2-D arrays of multiresponse ferromagnetic nanomagnets, submitted. 